Check for updates

Cite this: RSC Adv., 2019, 9, 29263

\title{
One-step deposition of antibacterial Ag@Pdop hybrid films on an NiTi alloy $\dagger$
}

\begin{abstract}
Yongkui Yin, ${ }^{\text {ab }}$ Ying Li, ${ }^{\text {bc }}$ Wei Cai ${ }^{a}$ and Jiehe Sui (iD *a
In this study, by one-step immersion, silver aggregates enwrapped in a polydopamine (Pdop) film (Ag@Pdop hybrid film) were deposited onto a nickel-titanium (NiTi) surface. The Ag aggregates in the Ag@Pdop hybrid films were formed through burst formation, following the Ostwald ripening and oriented attachment of $\mathrm{Ag}$ nanoparticles (NPs). As the coating time was increased, thickening of the Pdop film covered on the Ag aggregates could affect the antibacterial ability and biocompatibility of the AgaPdop hybrid films. When comprehensively comparing the experimental results, the film prepared for $12 \mathrm{~h}$ (Ag@12Pdop) exhibited excellent antibacterial activity against Staphylococcus aureus (S. aureus) and ideal cytocompatibility with human bone mesenchymal stem cells (hBMSCs). In addition, Ag@12Pdop exhibited better anticorrosion performance than polished NiTi in Hank's solution. Meanwhile, Ag@12Pdop showed sustainable Ag release and effectively inhibited the leaching of nickel (Ni) from the NiTi substrate. In this study, a simple method to fabricate Ag-containing films has been presented. The Ag@Pdop hybrid film may be able to significantly prevent infections induced by implantations.
\end{abstract}

Received 25th July 2019

Accepted 31st August 2019

DOI: $10.1039 / c 9 r a 05764 a$

rsc.li/rsc-advances designed to modify the surfaces of materials. Among the mentioned films, Ag-Pdop composite films can be fabricated by immersing a Pdop-decorated substrate in $\mathrm{AgNO}_{3}$ solution, and the $\mathrm{Ag}$ content can be regulated by regulating the $\mathrm{AgNO}_{3}$ concentration or its immersion time..$^{\mathbf{1 0 - 1 4}}$ In the meantime, the Pdop film can protect the substrate against corrosion in fluid environments. ${ }^{15,16}$ Therefore, Ag-Pdop composite films loaded with sufficient Ag NPs exhibit desirable antibacterial and anticorrosion performance. ${ }^{17}$ However, their further application has been limited by their multi-step and time-consuming manufacturing procedures. Thus, a simple route to fabricate Ag NPs containing Pdop films that exhibit desirable antibacterial performance and anticorrosion properties is required.

In this study, by a simple one-step immersion route in a mixture of $2 \mathrm{mg} \mathrm{mL} \mathrm{mL}^{-1}$ dopamine and $40 \mathrm{mM} \mathrm{AgNO}, \mathrm{Ag}$ aggregates enwrapped in Pdop (Ag@Pdop) hybrid films were deposited on NiTi alloys. The formation mechanism of the Ag@Pdop hybrid films was interpreted. The film prepared for $12 \mathrm{~h}$ (Ag@12Pdop) exhibited an excellent antibacterial ratio against Staphylococcus aureus (S. aureus) and ideal cytocompatibility with human bone mesenchymal stem cells (hBMSCs). Meanwhile, the sample exhibited better corrosion resistance than polished NiTi in Hank's solution. Furthermore, the film showed sustainable release of Ag ions and effectively inhibited leaching of Ni ion from NiTi alloy.

\section{Materials and methods}

\subsection{Materials and preparation methods}

$2 \mathrm{~mm}$ thick $\mathrm{Ni}_{50.8} \mathrm{Ti}_{49.2}($ at\%) plates with dimensions of $10 \mathrm{~mm} \times 10$ $\mathrm{mm}$ were used and were mirror polished using $1.5 \mu \mathrm{m}$ diamond 
paste. After that, the plates were successively cleaned in acetone, ethanol and deionized water and air-dried prior to preparation of the films. Ag@Pdop hybrid films were deposited on NiTi alloys by a simple one-step immersion approach. The coating solution was composed of $2 \mathrm{mg} \mathrm{mL}{ }^{-1}$ dopamine hydrochloride (Sigma-Aldrich) with or without $40 \mathrm{mM} \mathrm{AgNO}$, and the $\mathrm{pH}$ value was adjusted to 8.5 using Tris base (Sigma-Aldrich). ${ }^{18}$ The cleaned NiTi plates were directly immersed in $20 \mathrm{~mL}$ coating solution under constant vibration for $1,6,12,18$, or $24 \mathrm{~h}$ in a photophobic water bath constant temperature oscillator at $25^{\circ} \mathrm{C}$ (SHA-BA, Shanghai Guning Instrument Co., Ltd). The cyclotron amplitude was $20 \mathrm{~mm}$ and the rotation rate was controlled at $50 \mathrm{rpm}$. The immersion was performed only once. Finally, the obtained plates were ultrasonically cleaned in deionized water to remove weakly bound Pdop or $\mathrm{Ag} @$ Pdop and dried in vacuum at $40{ }^{\circ} \mathrm{C}$. To verify the consistency of the preparation, parallel experiments were carried out in triplicate. The specimens are termed Pdop and Ag@TPdop, where $T$ is the value of the coating time.

\subsection{Surface characterisation}

A scanning electron microscope (SEM; S4800, Hitachi, Japan) was used to examine the surface morphologies of the specimens. A transmission electron microscope (TEM; JEOL 2010, JEOL, Japan) was used to investigate the microstructures of the films. The chemical structures and compositions of the films were analyzed using Fourier transform infrared spectroscopy (Micro-FTIR, Thermo Fisher) and X-ray photoelectron spectroscopy (XPS, Bruker, USA), respectively. The Ag@6Pdop, Ag@12Pdop, and Ag@24Pdop hybrid films were scratched from the plates for TEM observation and FTIR testing. The topology structures and surface roughnesses of the samples were observed with an atomic force microscope (AFM, Bruker, USA) in tapping mode.

Contact angles were measured on an SL200B contact angle system (Kino, USA) at ambient humidity and temperature. A volume of $5 \mu \mathrm{L}$ of DI water was dropped on the sample. The data were collected in static mode in 5 different areas.

\subsection{Corrosion behavior and ion release}

The corrosion behavior of the samples was determined in Hank's solution (8.00 g L ${ }^{-1} \mathrm{NaCl}, 0.4 \mathrm{~g} \mathrm{~L}^{-1} \mathrm{KCl}, 0.06 \mathrm{~g} \mathrm{~L}^{-1} \mathrm{MgSO}_{4} \cdot 7 \mathrm{H}_{2} \mathrm{O}$, $0.06 \mathrm{~g} \mathrm{~L}^{-1} \mathrm{NaH}_{2} \mathrm{PO}_{4} \cdot 2 \mathrm{H}_{2} \mathrm{O}, 0.35 \mathrm{~g} \mathrm{~L}^{-1} \mathrm{NaHCO}_{3}, 1.0 \mathrm{~g} \mathrm{~L}^{-1}$ glucose, $0.6 \mathrm{~g} \mathrm{~L}^{-1} \mathrm{KH}_{2} \mathrm{PO}_{4}, 0.1 \mathrm{~g} \mathrm{~L}^{-1} \mathrm{MgCl} \cdot 6 \mathrm{H}_{2} \mathrm{O}$, and $0.14 \mathrm{~g} \mathrm{~L}^{-1} \mathrm{CaCl}_{2}$ at $\mathrm{pH}$ 7.4) and evaluated by a $\mathrm{CHI} 660 \mathrm{~A}$ electrochemical system $(\mathrm{CH}$ Instruments, USA) at $37{ }^{\circ} \mathrm{C}$. A saturated calomel electrode (SCE) was used as a reference, and a Pt foil served as the auxiliary electrode. The sample with an exposed area of $1 \mathrm{~cm}^{2}$ was maintained in the solution for $7200 \mathrm{~s}$ to obtain a steady open circuit potential (OCP). Then, the polarization scan started at $400 \mathrm{mV}$ below OCP and continued in the anodic direction with a scanning rate of $0.5 \mathrm{mV} \mathrm{s}^{-1}$.

Each sample was incubated in $5 \mathrm{~mL}$ of phosphate buffered saline solution (pH 7.2, PBS) at $37{ }^{\circ} \mathrm{C}$ to examine the release of $\mathrm{Ag}$ and $\mathrm{Ni}$ ions. The solutions were refreshed and collected at 1 , $4,7,14,21$, and 28 days. The cumulative concentration of released $\mathrm{Ag}$ and $\mathrm{Ni}$ ions was tested by inductively coupled plasma optical emission spectrometry (ICP-OES, Leeman, USA). Three samples were prepared for each experimental group.

\subsection{Evaluation of antibacterial activity}

The antibacterial activities of the samples were determined by bacterial counting using Staphylococcus aureus (S. aureus, ATCC 25923) bacteria cultured in Luria-Bertani (LB) medium. The polished NiTi and the modified NiTi were sterilized in $75 \%$ ethanol for 2 h. $50 \mu \mathrm{L}$ of bacterial suspension with a concentration of $10^{6} \mathrm{CFU} \mathrm{mL} \mathrm{m}^{-1}$ was placed on the sample in a 24 -well plate. The samples with bacterial solution were each covered with a sterilized polyethylene film $(1 \mathrm{~mm} \times 1 \mathrm{~mm})$ and incubated for $12 \mathrm{~h}$ at $37{ }^{\circ} \mathrm{C}$. After incubation, the samples and bacterial suspensions were transferred together into sterilized centrifuge tubes containing $5 \mathrm{~mL}$ PBS each and stirred by vortex for $5 \mathrm{~min}$ to dissociate the bacteria from the samples. After a series of dilutions, $100 \mu \mathrm{L}$ of each diluted solution was spread on solidified nutrient agar and incubated at $37^{\circ} \mathrm{C}$ for $18 \mathrm{~h}$. The visible colonies were counted, and the antibacterial ratio (AR) was calculated using the formula below:

$$
\mathrm{AR}=\frac{\mathrm{CFU}_{\text {control }}-\mathrm{CFU}_{\text {sample }}}{\mathrm{CFU}_{\text {control }}} \times 100 \%
$$

in which $\mathrm{CFU}_{\text {control }}$ is the average number of bacterial colonies on NiTi, and $\mathrm{CFU}_{\text {sample }}$ is the average number of bacterial colonies tested with the Ag@Pdop hybrid films.

\subsection{In vitro cytocompatibility evaluation}

Human bone mesenchymal stem cells (hBMSCs) (Stem Cell Bank, Chinese Academy of Science, Shanghai, China) were seeded on the surfaces of the samples to evaluate their cytocompatibility. The cells were first cultured in Dulbecco's modified Eagle's medium (DMEM, Gibco) supplemented with $10 \%$ fetal bovine serum and 1\% antimicrobial penicillin and streptomycin at $37{ }^{\circ} \mathrm{C}$ in a humid atmosphere of $5 \% \mathrm{CO}_{2}$. The culture medium was refreshed every two days until the cells became $90 \%$ confluent. Afterwards, the cells were washed twice with a PBS solution ( $\mathrm{pH} 7.4)$, detached by trypsinzation $(0.25 \%$ trypsin-EDTA), and resuspended in fresh media prior to seeding on the samples. $1 \mathrm{~mL}$ volumes of the cell suspension with a density of $5 \times 10^{4}$ cells per well were seeded in a 24 -well plate with the samples. After incubation for 1,2 , and 3 days, $50 \mu \mathrm{L}$ CCK-8 was injected into each well, and the plate was incubated for another $4 \mathrm{~h}$. Subsequently, $100 \mu \mathrm{L}$ of the resulting medium from each well was transferred to 96-well plates. In this study, DMEM was used as a negative control and DMEM with 10\% dimethylsulfoxide (DMSO) was used as a positive control. Parallel tests were conducted in triplicate. Then, the optical density (OD) value of the solution was measured using a microplate reader (Molecular Devices, M3, USA) at $570 \mathrm{~nm}$ with a reference wavelength of $630 \mathrm{~nm}$.

\section{Results and discussion}

\subsection{Composition and surface morphology}

The XPS spectra of the uncoated, Pdop-coated and Ag@Pdopcoated samples are shown in Fig. 1. Before the modification with Pdop, typical characteristic peaks of NiTi, such as $\mathrm{Ti}_{2 \mathrm{p}}, \mathrm{Ti}_{2 \mathrm{~s}}$, and $\mathrm{Ni}_{2 \mathrm{p}}$, were clearly observed. After the film was immersed in 
dopamine solution for $12 \mathrm{~h}$, the emergence of the signal of $\mathrm{N}_{1 \mathrm{~s}}$ centered at $399 \mathrm{eV}$, accompanied by the disappearance of the NiTi signals, and the enhanced signal of $\mathrm{C}_{1 \mathrm{~s}}$ suggested that a Pdop film was successfully covered on the NiTi alloy. When $\mathrm{AgNO}_{3}$ was added to the above solution, the characteristic Ag peaks emerged (Fig. 1a) in addition to the peaks of Pdop. Based on the high-resolution $\mathrm{Ag}_{3 \mathrm{~d}}$ XPS spectra, the bands centered at $368.15 \mathrm{eV}$ and $374.15 \mathrm{eV}$ belong to $\mathrm{Ag}_{3 \mathrm{~d}_{5 / 2}}$ and $\mathrm{Ag}_{3 \mathrm{~d}_{3 / 2}}$, respectively, revealing the presence of $\mathrm{Ag}(0)$ and the absence of other Ag compounds. ${ }^{17,19}$ Accordingly, the Ag@Pdop hybrid films were composed of metallic Ag and Pdop. Moreover, from the detailed elemental compositions listed in Table $\mathrm{S} 1 \dagger$ and Fig. $1 \mathrm{~b}$, the sample prepared for $6 \mathrm{~h}$ among the different coating times showed a maximum value of $\mathrm{Ag}$ atomic percentage $(0.84 \%)$.
The surface morphologies of the polished NiTi, Pdop, and Ag@Pdop are shown in Fig. 2. As shown in Fig. 2a, considerable grinding grooves exist on the NiTi surface, with a roughness of $2.92 \mathrm{~nm}$ (Fig. S1 $\dagger$ ). In contrast, the grooves of Pdop became blurry, indicating the presence of the Pdop film on the NiTi alloys (Fig. 2b). The surface roughness increased to $6.84 \mathrm{~nm}$ (Fig. S1 $\dagger$ ). Note that the addition of $\mathrm{AgNO}_{3}$ to the coating solution induced obvious variations of the surface morphology with increasing coating time. As shown in Fig. 2c and d, some aggregates with clear NPs were sparsely distributed on Ag@1Pdop. The average diameter of the aggregates was $233.26 \mathrm{~nm}$. As the coating time was prolonged to $6 \mathrm{~h}$, more aggregates with the same size as those on Ag@1Pdop were anchored on the surface (Fig. 2e and f). In comparison to Ag@1Pdop, the NPs were covered by a substance. When the coating time
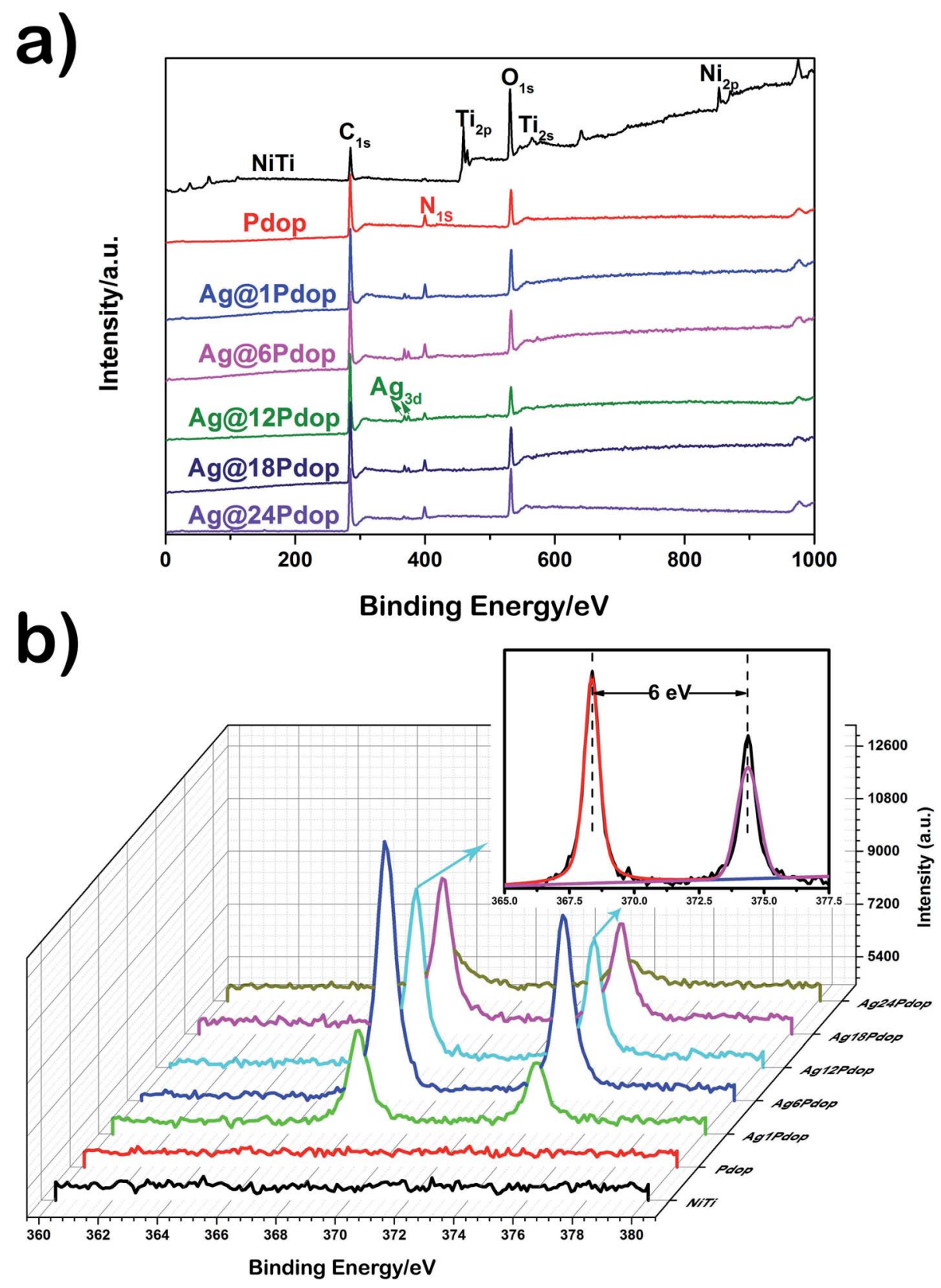

Fig. 1 XPS spectra of the polished NiTi, Pdop, and Ag@Pdop. (a) Full spectra and (b) high-resolution spectra of $\mathrm{Ag}_{3 \mathrm{~d}}$. 
was $12 \mathrm{~h}$, the aggregates with average sizes of $245.17 \mathrm{~nm}$ became swollen (Fig. $2 \mathrm{~g}$ and $\mathrm{h}$ ). The NPs become adherent and difficult to distinguish. When the coating time was prolonged to $18 \mathrm{~h}$ and even to $24 \mathrm{~h}$, the aggregates evolved into cloud-like chunks with average sizes of $258.4 \mathrm{~nm}$ and $289.26 \mathrm{~nm}$, respectively. No NPs could be detected in these stages (Fig. 2j-1). Meanwhile, the surface roughness increased as the coating time was prolonged, e.g. $19.6 \mathrm{~nm}$ for Ag@6Pdop, 27.8 nm for Ag@12Pdop, and 35.4 nm for Ag@24Pdop (Fig. S1†).

Fig. $\mathrm{S} 2 \dagger$ shows the EDAX spectra of the selected areas in Fig. 2. The major surface constituents of NiTi alloy were found to be $\mathrm{Ti}, \mathrm{Ni}, \mathrm{O}$, and $\mathrm{C}$. In contrast, in addition to these four elements, $\mathrm{N}$ element emerged in the spectrum of Pdopmodified NiTi. When $\mathrm{AgNO}_{3}$ was introduced, the $\mathrm{Ag}$ peaks also appeared. In addition, as the coating time increased, the content of $\mathrm{N}$ increased. However, beside the Ag@1Pdop (0.34 at $\%)$, the $\mathrm{Ag}$ content remained almost the same as the coating time increased.

Further information about the compositions and the structures of the Ag@Pdop hybrid films was obtained by FTIR and TEM analysis. The FTIR spectra of Pdop and Ag@Pdop are shown in Fig. S3. $\dagger$ For Pdop, the absorption peaks at $3435 \mathrm{~cm}^{-1}$, $1582 \mathrm{~cm}^{-1}$, and $1521 \mathrm{~cm}^{-1}$ belong to the phenolic hydroxyl stretching vibrations of catechol groups, stretch vibrations of aromatic rings, and $\mathrm{N}-\mathrm{H}$ shearing vibration of amide groups, respectively. ${ }^{17,19}$ In comparison, the functional groups in Ag@6Pdop, Ag@12Pdop and Ag@24Pdop were basically consistent with those of the Pdop. ${ }^{1719-21}$ However, the peaks of the phenolic hydroxyl stretching vibrations of the catechol groups and the stretch vibrations of the aromatic rings in the three Ag@Pdop hybrid films display red shifts to $3441 \mathrm{~cm}^{-1}$ and to $1627 \mathrm{~cm}^{-1}$, respectively, probably due to hydrogen bond and inductive effects between silver and phenolic hydroxyl groups. The TEM images of the Ag@6Pdop, Ag@12Pdop, and Ag@24Pdop hybrid films are given in Fig. 3. According to the HRTEM image of Ag@12Pdop, the lattice fringe of $0.24 \mathrm{~nm}$ can be indexed to the (111) facet of metallic Ag (Fig. 3c), suggesting that the dark particles were Ag NPs, while the surrounding gray substance should be a Pdop film (Fig. 3b and d). ${ }^{17}$ Moreover, Fig. 3b reveals that the Ag NPs of Ag@12Pdop were randomly embedded in the Pdop film. The $\mathrm{Ag}$ aggregates were not observed in Ag@6Pdop, whereas they could occasionally be
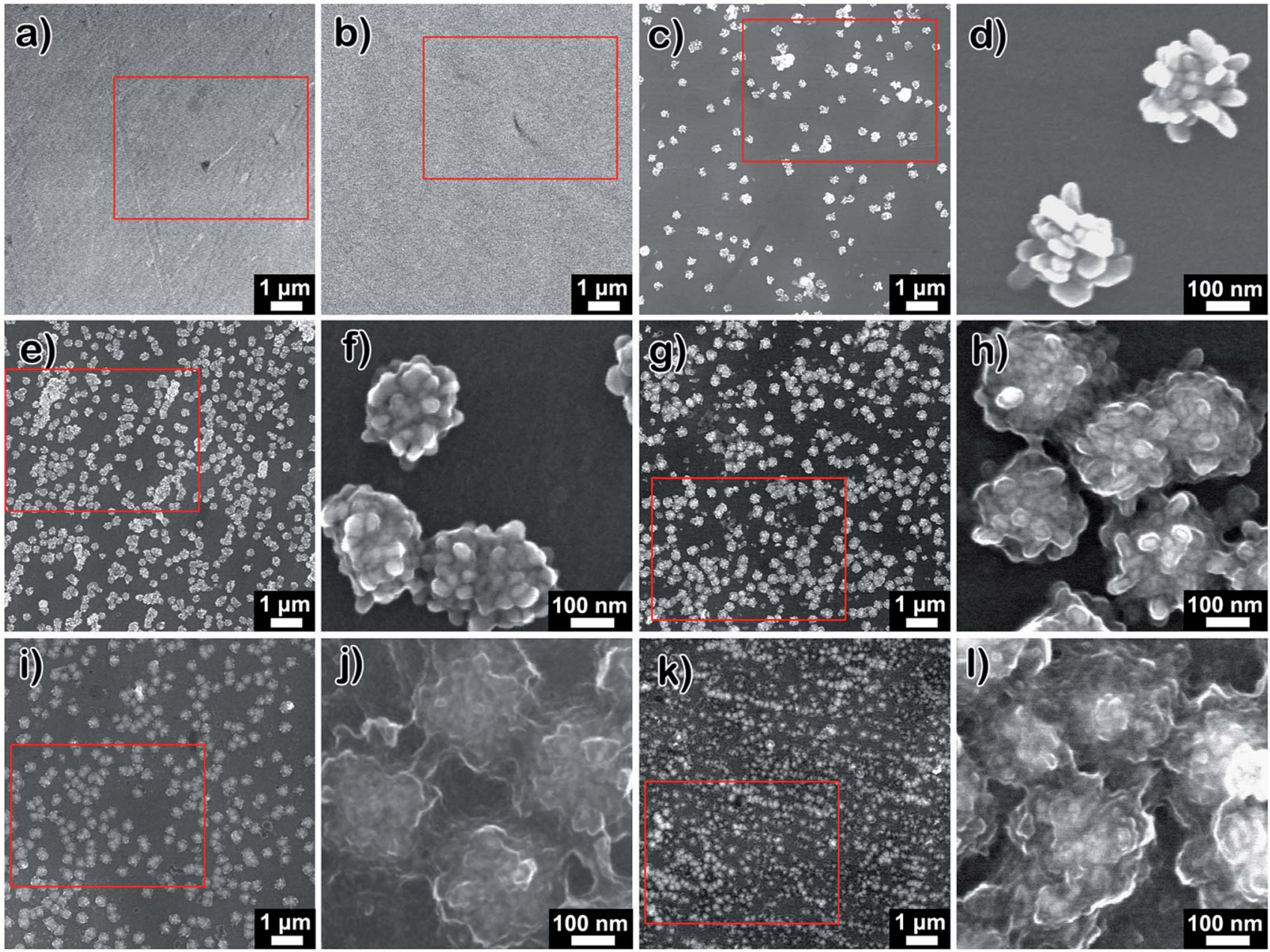

Fig. 2 Surface morphologies of polished NiTi, Pdop, and Ag@Pdop: (a) NiTi, (b) Pdop, (c and d) Ag@1Pdop, (e and f) Ag@6Pdop, (g and h) Ag@12Pdop, ( $i$ and j) Ag(18Pdop, and ( $k$ and l) Ag(24Pdop. The red rectangles are the selected areas for the EDAX analysis. 
found in Ag@12Pdop (blue circular region of Fig. 3b). Comparatively, considerable Ag aggregates enwrapped in thick Pdop films with an average thickness of $81.2 \mathrm{~nm}$ (blue circular region of Fig. 3d) can be observed in Ag@24Pdop. Additionally, the inhomogeneous ellipsoid-like Ag NPs existing in Ag@6Pdop with an average diameter of $13.82 \mathrm{~nm}$ evolved to homogeneous rice-like Ag NPs of Ag@24Pdop with an average size of $20.23 \mathrm{~nm}$ (Fig. S4†).

\subsection{Formation mechanism of a Ag@Pdop hybrid film}

Deposition of a Pdop film is considered to be a simple and flexible route for material functionalization, induced by $\mathrm{O}_{2}$ in the solution. ${ }^{17,22-25}$ In this study, Ag NPs could be instantaneously produced by the immediate reduction of $\mathrm{Ag}$ ions in dopamine solution due to the strong oxidation capacity $\left(E_{\mathrm{Ag}^{+} / \mathrm{Ag}}^{\theta}=+0.7996 \mathrm{~V}>E_{\mathrm{O}_{2} / \mathrm{OH}^{-}}^{\theta}=+0.401 \mathrm{~V}\right)$ and low solubility of $\mathrm{O}_{2}$. This process was driven by $\mathrm{Ag}$ ions, which consumed a portion of dopamine. To decrease the surface energy, the produced Ag NPs would self-assemble into Ag aggregates. Meanwhile, the excess dopamine polymerized and deposited on the surface of the NiTi alloy with $\mathrm{Ag}$ aggregates to form the $\mathrm{Ag} @$ Pdop hybrid film. This is a time-consuming process due to the low solubility of $\mathrm{O}_{2}$ in solution. For the different sedimentation rates, the maximum $\mathrm{Ag}$ aggregates could be achieved after coating for $6 \mathrm{~h}$, while the polymerization and deposition of Pdop still proceeded. This may explain why the density of the $\mathrm{Ag}$ aggregates increased from $1 \mathrm{~h}$ to $6 \mathrm{~h}$ and was maintained at the other time intervals. However, Ag@6Pdop displays the maximum Ag content detected by XPS. It has been demonstrated that the thickness of a Pdop film is time dependent and is close to the maximum value after coating for $24 \mathrm{~h}$ in a dopamine solution of $2 \mathrm{mg} \mathrm{mL}^{-1}, \mathrm{pH} \mathrm{8.5}$, and $\mathrm{O}_{2} \cdot{ }^{24,25}$ Thus, thickening the Pdop film from Ag@6Pdop to Ag@24Pdop decreased the Ag content, as ascertained by XPS. On the other hand, the thinner Pdop film in Ag@6Pdop or Ag@12Pdop could not withstand the integrality of the $\mathrm{Ag}$ aggregates, leading to large scale Ag NPs or scattered Ag aggregates distributed in the two scratched films (Fig. 3b and c). In contrast to Ag@6Pdop and Ag@12Pdop, large-scale perfect Ag@24Pdop composites can be observed in Fig. 3d. Additionally, the mean size and morphology of the Ag NPs in Ag@24Pdop are more uniform than those of Ag@6Pdop and Ag@12Pdop, probably due to the occurrence of Ostwald ripening and oriented attachment during the prolonged coating time.

Based on the above analysis, a proposed possible mechanism of Ag@Pdop hybrid film formation is illustrated in Fig. 4.
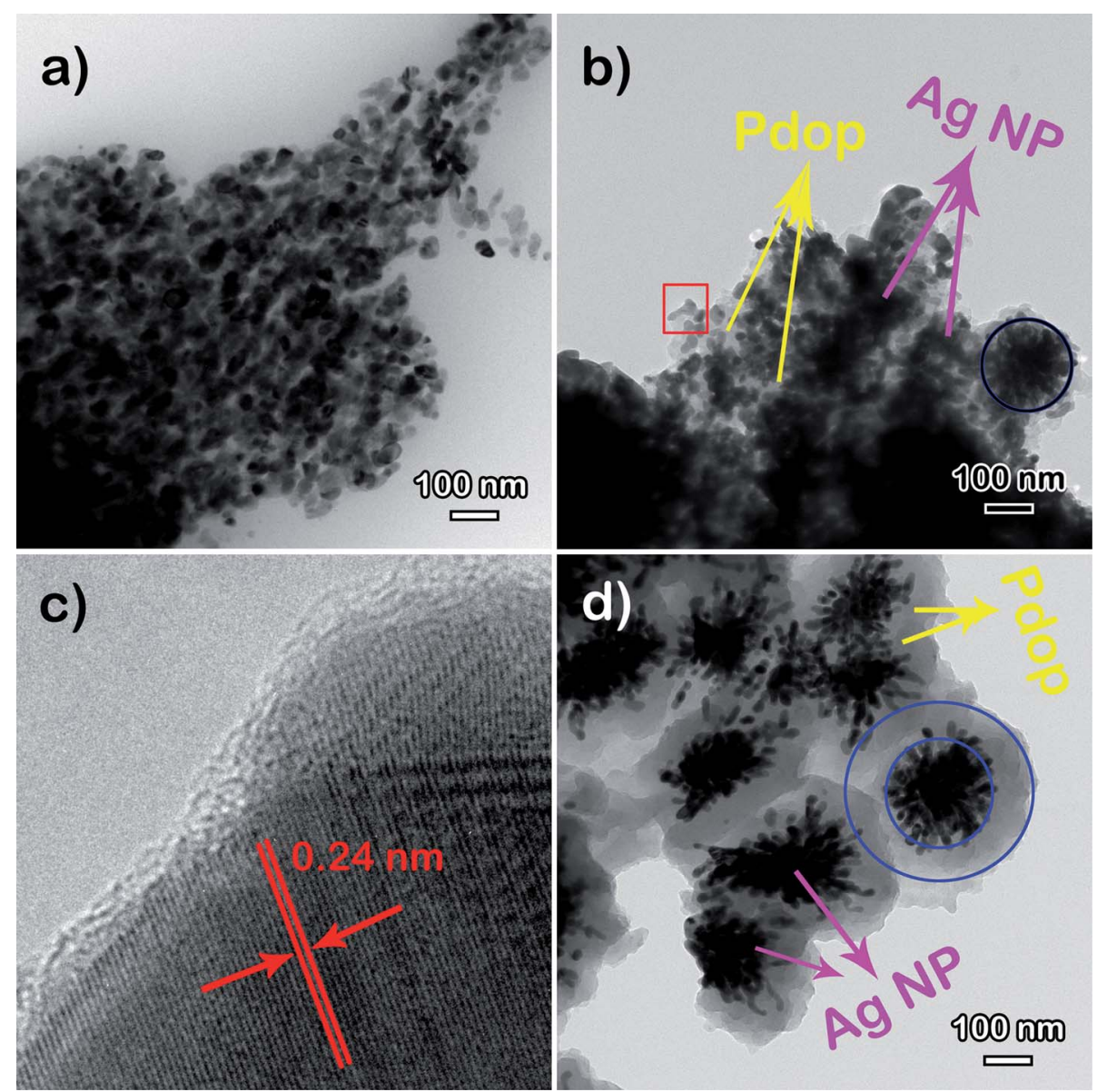

Fig. 3 TEM images of the Ag@Pdop hybrid films: (a) Ag@6Pdop and (b) Ag@12Pdop. (c) HRTEM images of the red region in (b) and of (d) Ag@24Pdop. 


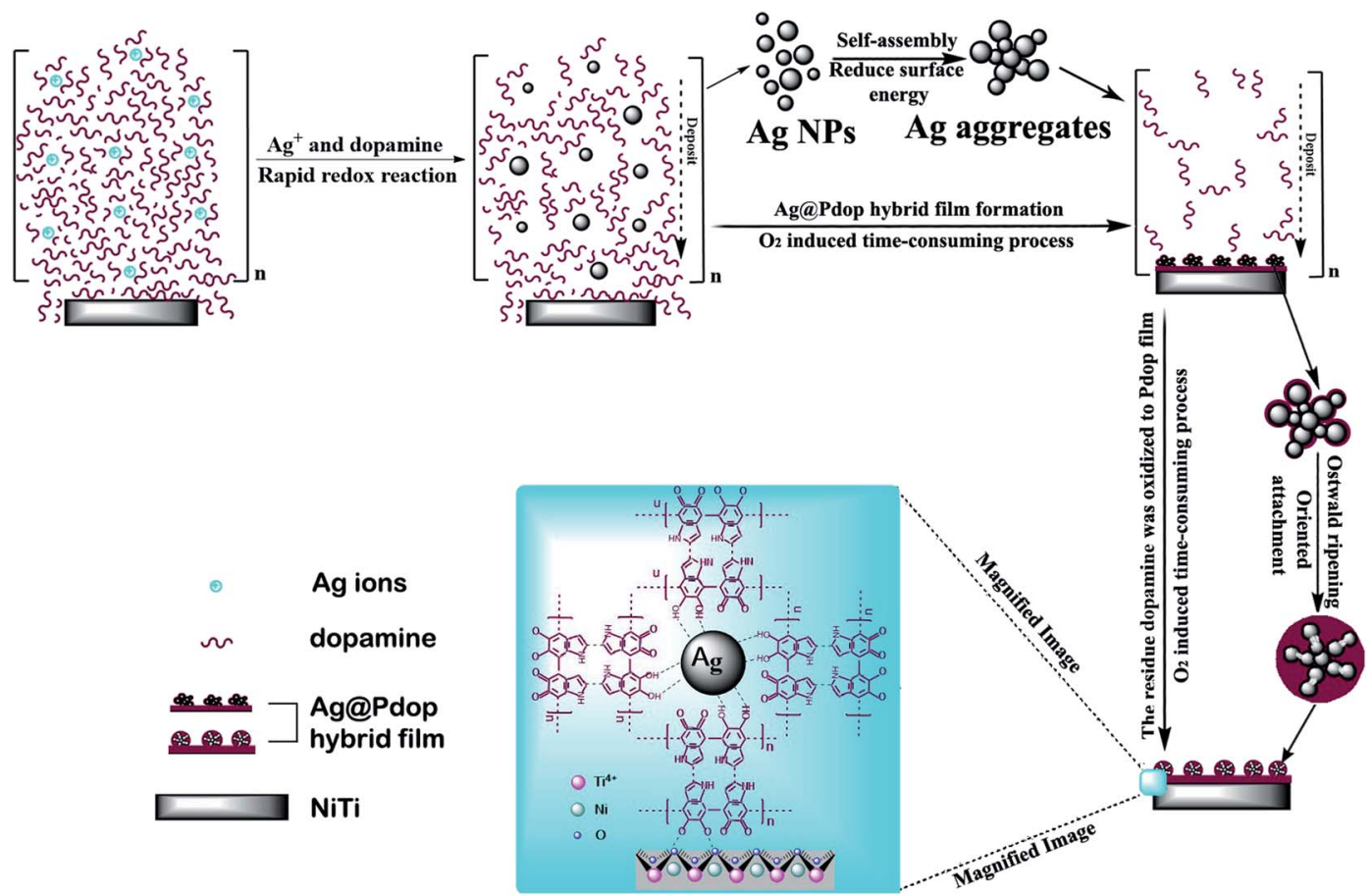

Fig. 4 Schematic of the formation mechanism of the Ag@Pdop hybrid film.

First, the Ag NPs were generated via a burst redox reaction between $\mathrm{Ag}$ ions and a portion of the dopamine and then selfassembled into $\mathrm{Ag}$ aggregates. Second, the residual dopamine polymerized to Pdop and deposited on the NiTi alloy with Ag aggregates to form the Ag@Pdop hybrid film. Third, a thickening Pdop film was further covered on the $\mathrm{Ag}$ aggregates as the coating time increased. In addition, oriented attachment and
Ostwald ripening caused the inhomogeneous Ag NPs to evolve into homogeneous rice-like Ag NPs.

\subsection{Corrosion resistance}

The polarization curves of the modified and unmodified NiTi alloys in Hank's solution are shown in Fig. 5. The polished NiTi exhibited the poorest corrosion behavior, with the lowest

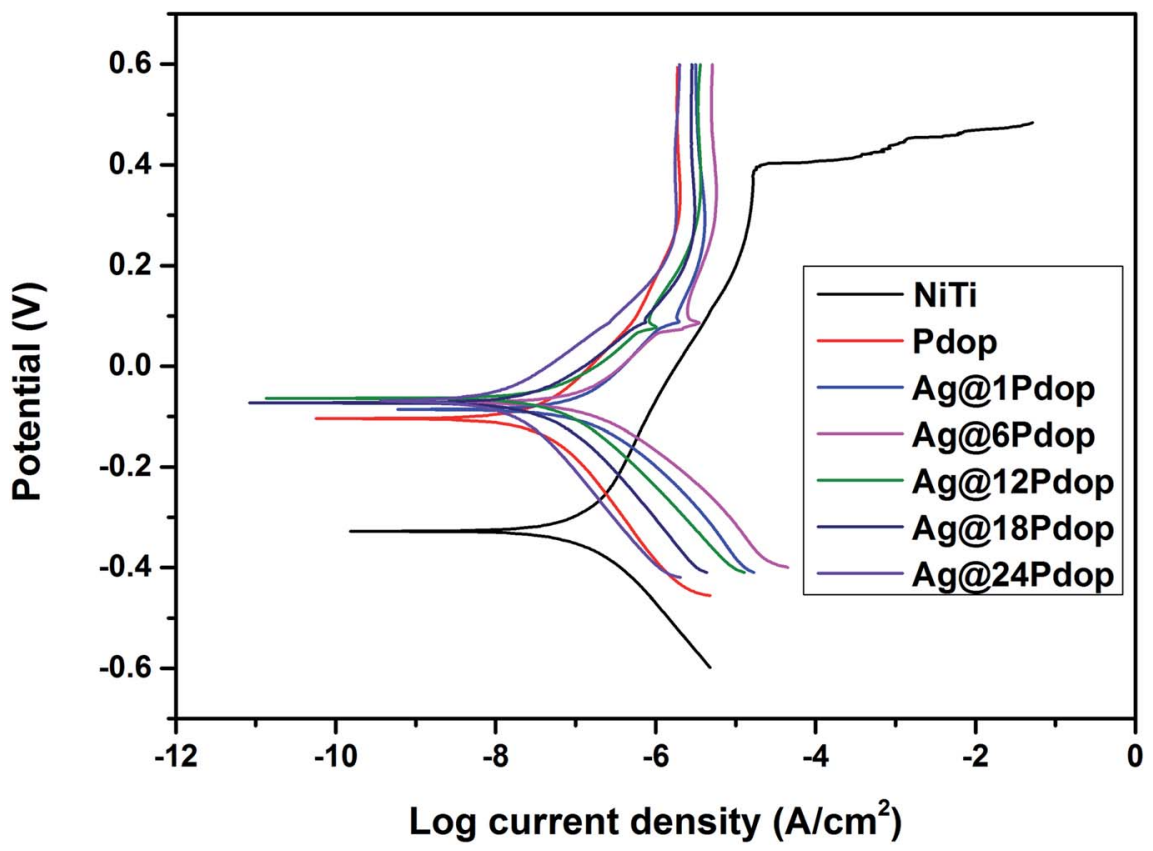

Fig. 5 Polarization curves of polished NiTi, Pdop and Ag@Pdop in Hank's solution. 


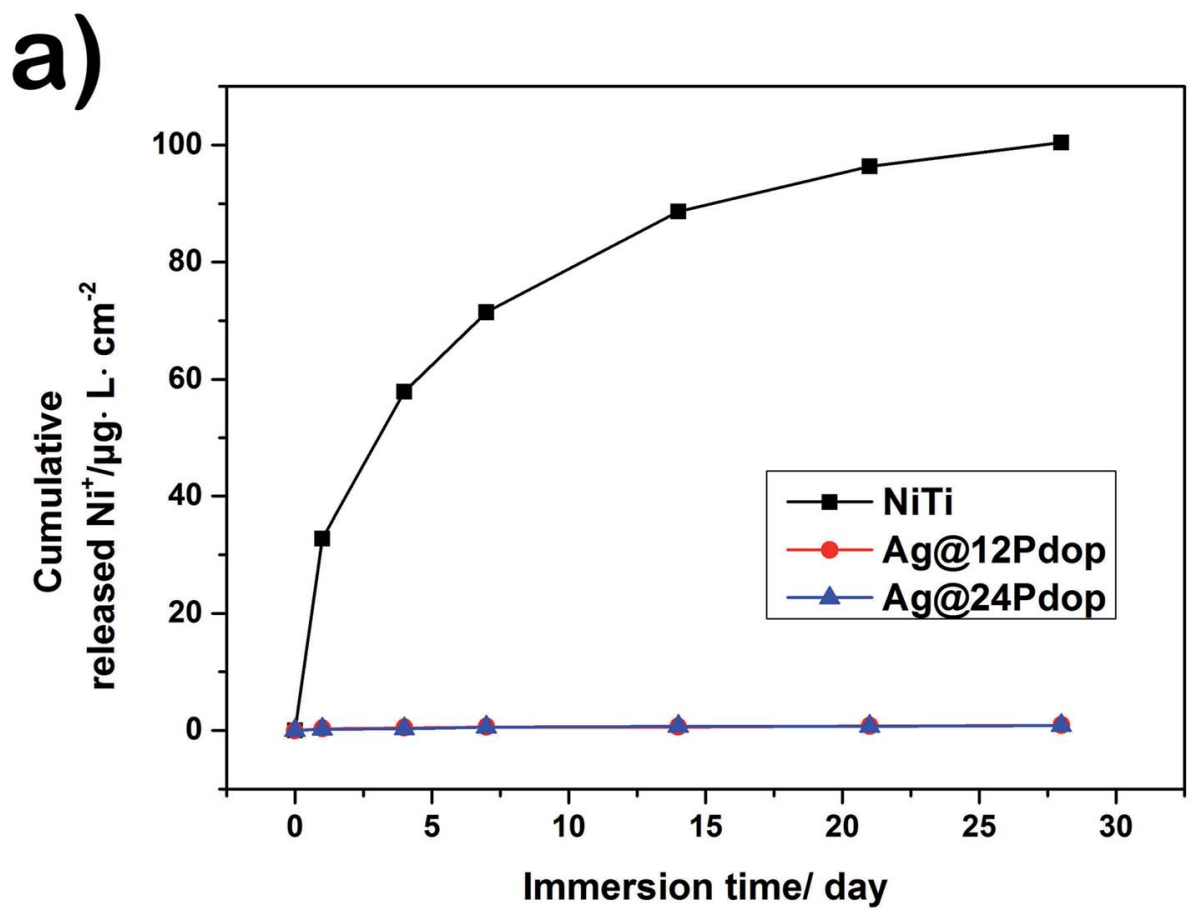

b)

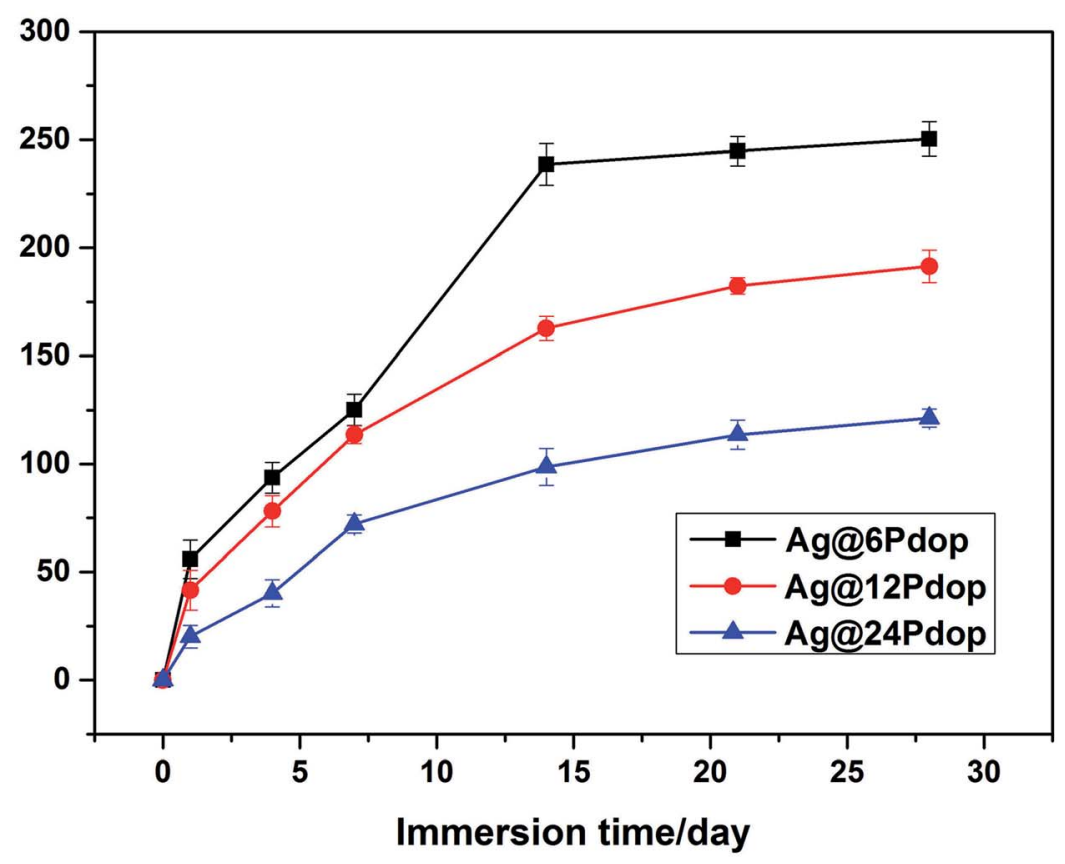

Fig. 6 Time-dependent release of $\mathrm{Ni}$ and $\mathrm{Ag}$ ions: (a) Ni ions; (b) Ag ions.

corrosion potential $\left(E_{\text {corr }}\right)$ of $-0.36 \mathrm{~V}$, the lowest breakdown potential $\left(E_{\mathrm{br}}\right)$ of $0.37 \mathrm{~V}$, and the highest corrosion current density $\left(I_{\text {corr }}\right)$ of $1.43 \times 10^{-7} \mathrm{~A} \mathrm{~cm}^{-1}$. Comparatively, the corrosion behavior was significantly enhanced, as evidenced by the $E_{\text {corr }}$ increment of $-0.09 \mathrm{~V}$ and $I_{\text {corr }}$ decrease of $2.07 \times 10^{-8}$ $\mathrm{A} \mathrm{cm}^{-1}$. After adding $\mathrm{AgNO}_{3}$ to the coating solution, $\mathrm{Ag} @$ Pdop exhibited a clear dependence on the immersion time. The $E_{\text {corr }}$, $I_{\text {corr }}$, and $E_{\mathrm{br}}$ values are listed in Table S3. $\dagger$ Enhanced $E_{\text {corr }}$ and decreased $I_{\text {corr }}$ were presented as the immersion time was prolonged. In addition, the minor peaks of $I_{\text {corr }}$ at about $0.09 \mathrm{~V}$ emerged in the curves of the Ag@Pdop-modified samples, which can be attributed to the dissolution of $\mathrm{Ag}$ during the process of polarization. ${ }^{11,17}$

\subsection{Release of $\mathrm{Ni}$ ions and $\mathrm{Ag}$ ions}

The release of Ni ions from the polished NiTi and Ag@12Pdop in PBS solution as a function of immersion time is expressed in 
Fig. 6a. It is suggested that the $\mathrm{Ni}$ ions leached continuously from the polished NiTi increased during the test. In contrast, the leaching content of $\mathrm{Ni}$ ions from $\mathrm{Ag@Pdop} \mathrm{was} \mathrm{almost}$ undetectable. Accordingly, Ag@Pdop hybrid films should be designed to remove the toxic effects of $\mathrm{Ni}$ ions leached from NiTi alloys.

The burst release of $\mathrm{Ag}$ ions is highlighted when applying $\mathrm{Ag}$ in the medical field; it may cause cell toxicity and significantly affect the antibacterial durability. ${ }^{17,26,27}$ The time dependence of $\mathrm{Ag}$ ion release from Ag@6Pdop, Ag@12Pdop, and Ag@24Pdop is shown in Fig. 6b. On the whole, the $\mathrm{Ag}$ release from Ag@6Pdop was terminated after immersion for 14 days. However, Ag@12Pdop and Ag@24Pdop showed constant Ag release that did not stop after 28 days. To be specific, the Ag release rates of Ag@6Pdop, Ag@12Pdop and Ag@24Pdop were about $2.33 \mu \mathrm{g} \mathrm{L}^{-1} \mathrm{~h}^{-1}, 1.53 \mu \mathrm{g} \mathrm{L}^{-1} \mathrm{~h}^{-1}$, and $0.84 \mu \mathrm{g} \mathrm{L}^{-1} \mathrm{~h}^{-1}$ for the first $24 \mathrm{~h}$, respectively, suggesting that the thickness of the Pdop film of Ag@Pdop is vital to control the Ag release rate. Moreover, the cumulative concentrations of $\mathrm{Ag}$ ions released from the samples during the testing time were nearly $230 \mu \mathrm{g}$ $\mathrm{L}^{-1}, 200 \mu \mathrm{g} \mathrm{L} \mathrm{L}^{-1}$, and $120 \mu \mathrm{g} \mathrm{L}^{-1}$, respectively; these are below the serum level of $300 \mu \mathrm{g} \mathrm{L} \mathrm{L}^{-1}$, which causes argyria, leukopenia, and alterations in renal, hepatic, and neural tissues. ${ }^{22}$ Meanwhile, the subsequent release of Ag ions would be diluted by the adjacent biological fluid when the samples were used in clinical applications. Thus, the designed Ag@Pdop hybrid films are expected to exhibit sustained antibacterial performance and lower the risk of cell toxicity.

\subsection{Antibacterial performance}

Staphylococcus aureus is a common pathogen that contributes to $5 \%$ to $40 \%$ of biomedical device-associated infection (BAI). Accordingly, colony forming of $S$. aureus was adopted to investigate the antibacterial performance. Typical photographs of the numbers of bacterial colonies on the polished NiTi and Ag@Pdop-modified NiTi are shown in Fig. 7. The highest bacterial counts can be ascertained on the NiTi alloy, while the colony number was significantly decreased $(P<0.001)$ on the Ag@Pdop-modified NiTi. To be specific, with reference to the control group (polished NiTi), the AR of Ag@6Pdop was above 99\%. The AR values of Ag@1Pdop, Ag@12Pdop, Ag@18Pdop, and Ag@24Pdop were 83.1\%, 96.3\%, 79.1\%, and 68.6\%, respectively. In contrast, large quantities of colonies were observed for the Pdop group, and they had no statistically significant difference from NiTi alloy $(P>0.05)$.

The accepted antibacterial mechanism of Ag NPs covers two aspects: one is that the $\mathrm{Ag}$ ions released from the $\mathrm{Ag}$ NPs can interact with thiol groups, inactivate bacterial proteins, and cause death of bacteria $;^{28}$ the other is that Ag NPs can bind to and penetrate bacterial cell walls, resulting in structural changes, degradation and destruction of the cells. ${ }^{29}$ In this study, the Ag aggregates were incorporated into Pdop films. The thicker Pdop film could not facilitate the release of $\mathrm{Ag}$ ions from Ag NPs, thereby hindering the interactions between the Ag NPs and bacteria. Thus, the prolonged coating time resulted in low AR for the Ag@Pdop hybrid films. Additionally, the weaker antibacterial performance of the film prepared for $1 \mathrm{~h}$ can be associated with the lower content of Ag loaded on Ag@1Pdop.

\subsection{In vitro cytotoxicity}

Although the cytotoxic levels of Ag NPs or silver ions were much higher than the antibacterial levels, cytotoxicity of several mammalian cell lines (e.g. rat liver cells (BRL 3A), male mouse germline cells (C18-4), human lung fibroblast cells and human glioblastoma cells (IMR-90)) was induced by Ag NPs, as has been reported. ${ }^{27,30-32}$ Thus, specific care should be taken before using $\mathrm{Ag}$ NPs as an antibacterial agent. The CCK-8 results of polished NiTi, Pdop, Ag@6Pdop, and Ag@12Pdop are shown in Fig. 8. Compared with the negative control, the cell viabilities of NiTi and Pdop slightly decreased and were maintained during the whole testing period (up to 3 days). However, Ag@6Pdop decreased the cell viability to $53 \%$ after incubation for $24 \mathrm{~h}$, which can be attributed to the cytotoxicity of the Ag NPs. As the incubating time was prolonged to 3 days, the cell viability of $\mathrm{Ag} @ 6$ Pdop even decreased to $18.9 \%$. It is noteworthy that the viabilities reached $81.7 \%, 86.3 \%$, and $89.6 \%$ after the cells were incubated with Ag@12Pdop for 1, 2, and 3 days, respectively. This viability is sufficient for clinical application.

Pdop-functionalized surfaces have been confirmed to exhibit ultralow cytotoxicity and excellent biocompatibility. ${ }^{20,33}$ It has been reported that Pdop-modified Ti can enhance cell adhesion and viability. ${ }^{34,35}$ In Ag@12Pdop, the Ag NPs are enwrapped in

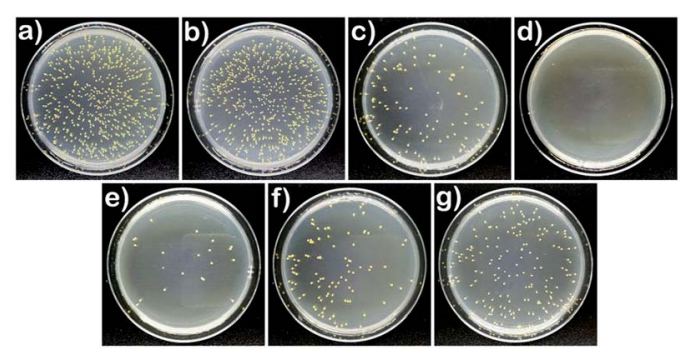

h)

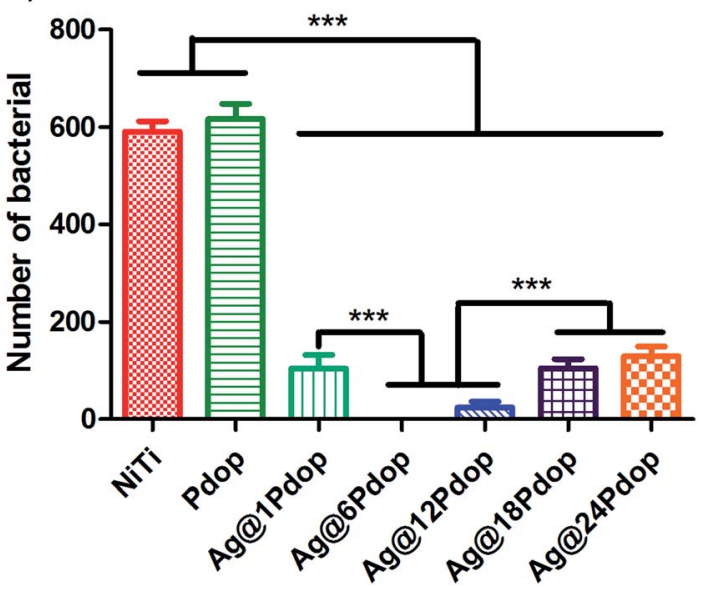

Fig. 7 Representative colony forming of bacteria treated with the samples: (a) NiTi, (b) Pdop, (c) Ag@1Pdop, (d) Ag@6Pdop, (e) Ag@12Pdop, (f) Ag@18Pdop, and (g) Ag@24Pdop. (h) Quantitative counts of bacteria treated with the samples. $* * *$ Represents $p<0.001$. 


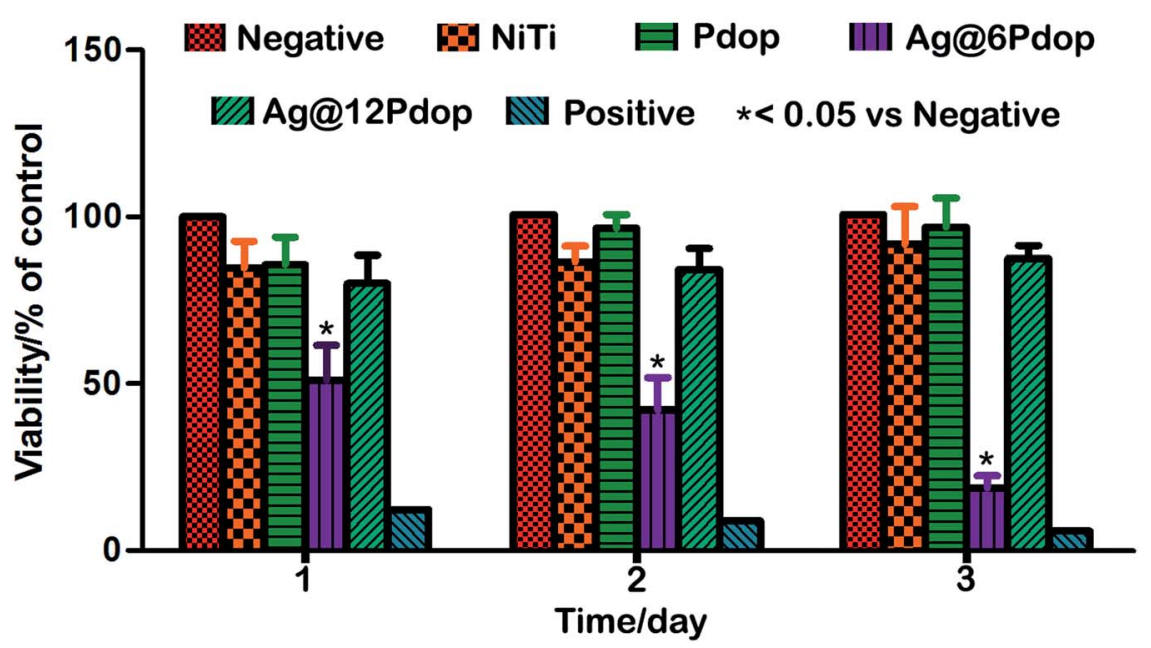

Fig. 8 Viabilities of hBMSCs cultured on NiTi, Pdop and Ag@Pdop-modified samples for different times.

the Pdop film to decrease the touch of the exposed Ag NPs. Accordingly, Ag@12Pdop exhibited satisfactory cell viability. In contrast, the notable cell toxicity of Ag@6Pdop can be attributed to the thinner Pdop film. Moreover, the increment of the surface roughness and hydrophilicity of Ag@12Pdop may help enhance cell adhesion and proliferation (Fig. S1 $\dagger$ ).

\section{Conclusion}

In this study, a series of Ag@Pdop hybrid films were deposited on NiTi alloys through a one-step immersion method. The thickness of the Pdop film in Ag@Pdop could be controlled by tailoring the coating time. The thickness of the Pdop films could affect the corrosion resistance properties, antibacterial performance, and biocompatibility of the fabricated Ag@Pdop. In particular, Ag@12Pdop exhibited excellent antibacterial performance, anticorrosion properties, and sustainable release of $\mathrm{Ag}$ ions. Additionally, the film could effectively prevent leaching of $\mathrm{Ni}$ ions from NiTi. This is considered to be a simple way to fabricate Ag-containing films and to further expand the applications of NiTi alloys.

\section{Conflicts of interest}

We declare that we do not have any commercial or associative interest that represents a conflict of interest in connection with the work submitted.

\section{Acknowledgements}

This work was supported by the National Natural Science Foundation of China (No. 51622101, 51771065, and 51871082), Fundamental Research Operating Expenses of Provincial Colleges and Universities in Heilongjiang Province (2017KYYWFMY0703), and Mudanjiang Instructional Science and Technology Project (Z2017s0065).

\section{References}

1 S. A. Shabalovskaya, On the nature of the biocompatibility and on medical applications of NiTi shape memory and superelastic alloys, Bio-Med. Mater. Eng., 1996, 6, 267-274.

2 H. Funakubo, Shape memory alloys, Gordon and Breach Science Publishers, New York, 1987, pp. 226-269.

3 D. H. Wang, N. J. Ge, S. Qian, J. H. Li, Y. Q. Qiao and X. Y. Liu, Selenium doped Ni-Ti layered double hydroxide (Ni-Ti LDH) films with selective inhibition effect to cancer cells and bacteria, RSC Adv., 2015, 5, 106848-106859.

4 C. H. Zhao, S. Pandit, Y. F. Fu, I. Mijakovic, A. Jesorka and J. H. Liu, Graphene oxide based coatings on nitinol for biomedical implant applications: effectively promote mammalian cell growth but kill bacteria, RSC Adv., 2016, 6, 38124-38134.

5 M. Cempel and G. Nikel, Nickel: a review of its sources and environmental toxicology, Pol. J. Environ. Stud., 2006, 15, 375-384.

6 R. A. Ahmed, S. A. Fadl-allah, N. El-Bagoury and S. M. F. G. ElRabf, Improvement of corrosion resistance and antibacterial effect of NiTi orthopedic materials by chitosan and gold nanoparticles, Appl. Surf. Sci., 2014, 292, 390-399.

7 M. J. Li, C. Schlaich, M. W. Kulka, I. S. Donskyi, T. Schwerdtle, W. E. S. Unger and R. Haag, Mussel-inspired coatings with tunable wettability, for enhanced antibacterial efficiency and reduced bacterial adhesion, $J$. Mater. Chem. B, 2019, 7, 3438-3445.

8 L. L. Yin, Z. X. Fu, Y. Li, B. Liu, Z. J. Lin, J. Y. Lu, X. Chen, X. P. Han, Y. D. Deng, W. B. Hu, D. R. Zou and C. Zhong, Enhanced antibacterial properties of biocompatible titanium via electrochemically deposited $\mathrm{Ag} / \mathrm{TiO}_{2}$ nanotubes and chitosan-gelatin-Ag-ZnO complex coating, RSC Adv., 2019, 9, 4521-4529.

9 X. J. Wan, L. S. Wu, H. Pei, H. Q. Ke, G. H. Yang and J. N. Tang, Surface functionality density regulated in situ reduction of nanosilver on hierarchial wrinkled 
mesoporous silica nanoparticles and their antibacterial activity, RSC Adv., 2018, 8, 19420-19425.

10 S. Sharma, P. Sanpui, A. Chattopadhyay and S. S. Ghosh, Fabrication of antibacterial silver nanoparticle-sodium alginate-chitosan composite films, RSC Adv., 2012, 2, 58375843.

11 T. Fu, Y. G. Shen, Z. Alajmi, Y. N. Wang, S. Y. Yang and G. Li, Sol-gel derived Ag-containing $\mathrm{TiO}_{2}$ films on surface roughened biomedical NiTi alloy, Ceram. Int., 2014, 40, 12423-12429.

12 X. M. Zhang, Z. Y. Li, X. B. Yuan, Z. D. Cui, H. J. Bao, X. Li, Y. D. Liu and X. J. Yang, Cytotoxicity and antibacterial property of titanium alloy coated with silver nanoparticlecontaining polyelectrolyte multilayer, Mater. Sci. Eng., C, 2013, 33, 2816-2820.

13 H. Y. Luo, C. W. Gu, W. H. Zheng, F. Dai, X. L. Wang and Z. Zheng, Facile synthesis of novel size-controlled antibacterial hybrid spheres using silver nanoparticles loaded with poly-dopamine spheres, RSC Adv., 2015, 5, 13470-13477.

14 J. L. Chen, M. L. Mei, Q. L. Li and C. H. Chu, Mussel-inspired silver-nanoparticle coating on porous titanium surfaces to promote mineralization, RSC Adv., 2016, 6, 104025-104035.

15 L. Jiang, G. C. Jin, J. Kang, L. M. Yu, W. Yoon, M. Lim, K. I. Par, M. Lee and D. C. Jin, Surface characteristics of mussel-inspired polydopamine coating on titanium substrates, J. Wuhan Univ. Technol., Mater. Sci. Ed., 2014, 29, 197-200.

16 F. Singer, M. Schlesak, C. Mebert, S. Höhn and S. Virtanen, Corrosion properties of polydopamine coatings formed in one-Step immersion process on magnesium, ACS Appl. Mater. Interfaces, 2015, 7, 26758-26766.

17 Z. J. Jia, P. Xiu, M. Li, X. C. Xu, Y. Y. Shi, Y. Cheng, S. C. Wei, Y. F. Zheng, T. F. Xi, H. Cai and Z. J. Liu, Bioinspired anchoring AgNPs onto micro-nanoporous $\mathrm{TiO}_{2}$ orthopedic coatings: trap-killing of bacteria, surface-regulated osteoblast functions and host responses, Biomaterials, 2016, 75, 203-222.

18 L. P. Yang, J. H. Kong, D. Zhou, J. M. Ang, S. L. Phua, W. A. Yee, H. Liu, Y. Z. Huang and X. H. Lu, Transitionmetal-ion-mediated polymerization of dopamine: MusselInspired approach for the facile synthesis of robust transition-metal nanoparticle-graphene hybrids, Chem.Eur. J., 2014, 20, 7776-7783.

19 T. S. Sileika, H. D. Kim, P. Maniak and P. B. Messersmith, Antibacterial performance of polydopamine-modified polymer surfaces containing passive and active components, ACS Appl. Mater. Interfaces, 2011, 3, 4602-4610.

20 Q. Yue, M. H. Wang, Z. K. Sun, C. Wang, C. Wang, Y. H. Deng and D. Y. Zhao, A versatile ethanol-mediated polymerization of dopamine for efficient surface modification and the construction of functional core-shell nanostructures, $J$. Mater. Chem. B, 2013, 1, 6085-6093.

21 S. Q. Xiong, Y. Wang, J. Zhu, J. R. Yu and Z. M. Hu, Musseladhesive-inspired fabrication of multifunctional silver nanoparticle assemblies, Langmuir, 2015, 31, 5504-5512.
22 M. Li, Q. Liu, Z. J. Jia, X. C. Xu, Y. Y. Shi, Y. Cheng and Y. F. Zheng, Polydopamine-induced nanocomposite Ag/CaP coatings on the surface of titania nanotubes for antibacterial and osteointegration functions, J. Mater. Chem. B, 2015, 3, 8796-8805.

23 M. S. Akin, M. Yilmaz, E. Babur, B. Ozdemir, H. Erdogan, U. Tamer and G. Demirel, Large area uniform deposition of silver nanoparticles through bio-inspired polydopamine coating on silicon nanowire arrays for practical SERS applications, J. Mater. Chem. B, 2014, 2, 4894-4900.

24 N. F. Della Vecchia, A. Luchini, A. Napolitano, G. D'Errico, G. Vitiello, N. Szekely, M. d'Ischia and L. Paduano, Tris buffer modulates polydopamine growth, aggregation, and paramagnetic properties, Langmuir, 2014, 30, 9811-9818.

25 F. Bernsmann, V. Ball, F. Addiego, A. Ponche, M. Michel, J. J. D. Gracio, V. Toniazzo and D. Ruch, Dopaminemelanin film deposition depends on the used oxidant and buffer solution, Langmuir, 2011, 27, 2819-2825.

26 P. Dallas, V. K. Sharma and R. Zboril, Silver polymeric nanocomposites as advanced antimicrobial agents: classification, synthetic paths, applications, and perspectives, Adv. Colloid Interface Sci., 2011, 166, 119-135.

27 L. Y. Guo, W. Y. Yuan, Z. S. Lu and C. M. Li, Polymer/ nanosilver composite coatings for antibacterial applications, Colloids Surf., A, 2013, 439, 69-83.

28 W. K. Jung, H. C. Koo, K. W. Kim, S. Shin, S. H. Kim and Y. H. Park, Antibacterial activity and mechanism of action of the silver ion in Staphylococcus aureus and Escherichia coli, Appl. Environ. Microbiol., 2008, 74, 2171-2178.

29 I. Sondi and B. Salopek-Sondi, Silver nanoparticles as antimicrobial agent: a case study on E coli as a model for gram-negative bacteria, J. Colloid Interface Sci., 2004, 275, 177-182.

30 S. M. Hussain, K. L. Hess, J. M. Gearhart, K. T. Geiss and J. J. Schlager, In vitro toxicity of nanoparticles in BRL 3A rat liver cells, Toxicol. In Vitro, 2005, 19, 975-983.

31 R. Foldbjerg, P. Olesen, M. Hougaard, D. A. Dang, H. J. Hoffmann and H. Autrup, PVP-coated silver nanoparticles and silver ions induce reactive oxygen species, apoptosis and necrosis in THP-1 monocytes, Toxicol. Lett., 2009, 190, 156-162.

32 P. V. AshaRani, G. L. K Mun, M. P. Hande and S. Valiyaveettil, Cytotoxicity and genotoxicity of silver nanoparticles in human cells, ACS Nano, 2009, 3, 279-290.

33 M. Lai, K. Y. Cai, L. Zhao, X. Y. Chen, Y. H. Hou and Z. X. Yang, Surface Functionalization of $\mathrm{TiO}_{2}$ Nanotubes with bone morphogenetic protein 2 and its synergistic effect on the differentiation of mesenchymal stem cells, Biomacromolecules, 2011, 12, 1097-1105.

34 J. Cha, Y. Peng, Z. Zheng, P. Y. Sun and X. L. Wang, Silverreleasing and antibacterial activities of polyphenol-based polyurethanes, J. Appl. Polym. Sci., 2015, 1, 41349.

35 M. Yu, J. Hwang and T. J. Deming, Role of L-3,4dihydroxyphenylalanine in mussel adhesive proteins, J. Am. Chem. Soc., 1999, 121, 5825-5826. 